\title{
SINGULAR HYPERBOLICITY AND SECTIONAL LYAPUNOV EXPONENTS OF VARIOUS ORDERS
}

\author{
LUCIANA SALGADO
}

\begin{abstract}
It is given notions of singular hyperbolicity and sectional Lyapunov exponents of orders beyond the classical ones, namely, other dimensions besides the dimension 2 and the full dimension of the central subbundle of the singular hyperbolic set. It is obtained a characterization of dominated splittings, partial and singular hyperbolicity in this broad sense, by using Lyapunov exponents and the notion of infinitesimal Lyapunov functions. Furthermore, it is given alternative requirements to obtain singular hyperbolicity. As an application we obtain some results related to singular hyperbolic sets for flows.
\end{abstract}

\section{INTRODUCTION AND STATEMENT OF RESULTS}

Let $M$ be a compact $C^{\infty}$ riemannian $n$-dimensional manifold, $n \geq 3$. Let $\mathfrak{X}^{1}(M)$ the set of $C^{1}$ vector fields on $M$, endowed with the $C^{1}$ topology. And denote by $X_{t}: M \rightarrow M$ the $C^{1}$ flow generated by $X$.

In a remarkable work Morales, Pacífico and Pujals [17] defined the so called singular hyperbolic systems, in order to describe the behaviour of Lorenz attractor. It is an extension of the hyperbolic theory for invariant sets for flows which are not (uniformly) hyperbolic, but which have some robust properties, certain kind of weaker hyperbolicity and also admit singularities. In [18], the same authors proved that every robustly transitive singular set for a three dimensional flow is a partially hyperbolic attractor or repeller and the singularities in this set must be Lorenz-like.

In this paper, we prove a relation between the $\mathcal{J}$-algebra of Potapov $[20,21,25]$ a new definition of singular hyperbolicity, envolving intermediate dimensions of the central subbundle.

The $\mathcal{J}$-algebra here means a pseudo-euclidean structure given by $C^{1}$ non-degenerate quadratic form $\mathcal{J}$, defined on $\Lambda$, which generates positive and negative cones with maximal dimension $p$ and $q$, respectively.

The maximal dimension of a cone in $T_{x} M$ is the maximal dimension of the subspaces contained in there.

We are going to prove sufficient and necessary conditions for a flow to be singular hyperbolic of some order, in a sense to be clarified below.

2000 Mathematics Subject Classification. Primary: 37D30; Secondary: 37D25.

Key words and phrases. Dominated splitting, partial hyperbolicity, sectional hyperbolicity, Lyapunov function.

L.S. is partially supported by a Fapesb-JCB0053/2013, PRODOC-UFBA2014, CNPq postdoctoral schoolarship at Universidade Federal do Rio de Janeiro. She also thanks to A. Hammerlindl for fruitful conversations during International Conference Dynamics Beyond Uniform Hyperbolicity - Provo-UT 2017. 
It is also given a characterization of singular and sectional hyperbolicity for a flow over a compact invariant set, improving a result in [1].

The text is organized as follow. In first section, it is given the main definitions and stated the results. In second section, it is presented the main tools by using the notion of $\mathcal{J}$-algebra of Potapov. In third section, it is proved the main theorems.

\subsection{Preliminary definitions and Main results.}

Before presenting the main statements, we give some definitions.

Let $M$ be a connected compact finite $n$-dimensional manifold, $n \geq 3$, with or without boundary. We consider a vector field $X$, such that $X$ is inwardly transverse to the boundary $\partial M$, if $\partial M \neq \emptyset$. The flow generated by $X$ is denoted by $X_{t}$.

An invariant set $\Lambda$ for the flow of $X$ is a subset of $M$ which satisfies $X_{t}(\Lambda)=\Lambda$ for all $t \in \mathbb{R}$. The maximal invariant set of the flow is $M(X):=\cap_{t \geq 0} X_{t}(M)$, which is clearly a compact invariant set.

A singularity for the vector field $X$ is a point $\sigma \in M$ such that $X(\sigma)=0$ or, equivalently, $X_{t}(\sigma)=\sigma$ for all $t \in \mathbb{R}$. The set formed by singularities is the singular set of $X$ denoted $\operatorname{Sing}(X)$ and $\operatorname{Per}(X)$ is the set of periodic points of $X$. We say that a singularity is hyperbolic if the eigenvalues of the derivative $D X(\sigma)$ of the vector field at the singularity $\sigma$ have nonzero real part. The set of critical elements of $X$ is the union of the singularities and the periodic orbits of $X$, and will be denoted by $\operatorname{Crit}(X)$.

We recall that a hyperbolic set for a flow $X_{t}$ is an invariant subset of $\Lambda \subset M$ with a decomposition $T_{\Lambda} M=E^{s} \oplus E^{X} \oplus E^{u}$ of the tangent bundle which is a continuous splitting, where $E^{X}$ is the direction of the vector field, the subbundles are invariant under the derivative $D X_{t}$ of the flow

$$
D X_{t} \cdot E_{x}^{*}=E_{X_{t}(x)}^{*}, \quad x \in \Lambda, \quad t \in \mathbb{R}, \quad *=s, X, u ;
$$

$E^{s}$ is uniformly contracted by $D X_{t}$ and $E^{u}$ is uniformly expanded: there are $K, \lambda>0$ so that

$$
\left\|\left.D X_{t}\right|_{E_{x}^{s}}\right\| \leq K e^{-\lambda t}, \quad\left\|\left.D X_{-t}\right|_{E_{x}^{u}}\right\| \leq K e^{-\lambda t}, \quad x \in \Lambda, \quad t \in \mathbb{R} .
$$

Recall that the index of a hyperbolic periodic orbit of a flow is the dimension of the contracting subbundle of its hyperbolic splitting.

Our main results is the following.

Let $\Lambda \subset M$ be a compact invariant subset for $X$.

Definition 1. A dominated splitting over a compact invariant set $\Lambda$ of $X$ is a continuous $D X_{t}$ invariant splitting $T_{\Lambda} M=E \oplus F$ with $E_{x} \neq\{0\}, F_{x} \neq\{0\}$ for every $x \in \Lambda$ and such that there are positive constants $K, \lambda$ satisfying

$$
\left\|\left.D X_{t}\right|_{E_{x}}\right\| \cdot\left\|\left.D X_{-t}\right|_{F_{X_{t}(x)}}\right\|<K e^{-\lambda t} \text {, for all } x \in \Lambda, \text { and all } t>0 .
$$

A compact invariant set $\Lambda$ is said to be partially hyperbolic if it exhibits a dominated splitting $T_{\Lambda} M=E \oplus F$ such that subbundle $E$ is uniformly contracted. In this case $F$ is the central subbundle of $\Lambda$. 
A compact invariant set $\Lambda$ is said to be singular-hyperbolic if it is partially hyperbolic and the action of the tangent cocycle expands volume along the central subbundle, i.e.,

$$
\left|\operatorname{det}\left(\left.D X_{t}\right|_{F_{x}}\right)\right|>C e^{\lambda t}, \forall t>0, \forall x \in \Lambda \text {. }
$$

The following definition was given as a particular case of singular hyperbolicity.

Definition 2. A sectional hyperbolic set is a singular hyperbolic one such that for every twodimensional linear subspace $L_{x} \subset F_{x}$ one has

$$
\left|\operatorname{det}\left(\left.D X_{t}\right|_{L_{x}}\right)\right|>C e^{\lambda t}, \forall t>0 \text {. }
$$

\subsection{Singular hyperbolicity of various orders.}

Given $E$ a vector space, we denote by $\wedge^{p} E$ the exterior power of order $p$ of $E$, defined as follows. If $v_{1}, \ldots, v_{n}$ is a basis of $E$ then $\wedge^{p} E$ is generated by $\left\{v_{i_{1}} \wedge \cdots \wedge v_{i_{p}}\right\}_{1 \leq i \leq n, i_{j} \neq i_{k}, j \neq k}$. Any linear transformation $A: E \rightarrow F$ induces a transformation $\wedge^{p} A: \wedge^{p} E \rightarrow \wedge^{p} F$. Moreover, $v_{i_{1}} \wedge \cdots \wedge v_{i_{p}}$ can be viewed as the $p$-plane generated by $\left\{v_{i_{1}}, \cdots, v_{i_{p}}\right\}$ if $i_{j} \neq i_{k}, j \neq k$. As a reference for more information about exterior powers it is recommended [7], for instance.

We may define a new kind of singular hyperbolicity.

Definition 3. A compact invariant set $\Lambda$ is $p$-singular hyperbolic (or $p$-sectionally hyperbolic) for a $C^{1}$ flow $X$ if there exists a partially hyperbolic splitting $T_{\Lambda} M=E \oplus F$ such that $E$ is uniformly contracting and the central subbundle $F$ is $p$-sectionally expanding, with $2 \leq p \leq \operatorname{dim}(F)$.

If $L_{x}$ is a $p$-plane, we can see it as $\widetilde{v} \in \wedge^{p}\left(F_{x}\right) \backslash\{0\}$ of norm one. Hence, to obtain the singular expansion we just need to show that for some $\lambda>0$ and every $t>0$ holds the following inequality

$$
\left\|\wedge^{p} D X_{t}(x) \cdot \widetilde{v}\right\|>C e^{\lambda t} .
$$

Our first main result concerns in a characterization of singular hyperbolicity of any order via infinitesimal Lyapunov functions, following [1], [2], [11], [21], [23], [25].

Recall that, if $T: Z \rightarrow Z$ is a measurable map, we say that a probability measure $\mu$ is an invariant measure of $T$, if $\mu\left(T^{-1}(A)\right)=\mu(A)$, for every measurable set $A \subset Z$. We say that $\mu$ is an invariant measure of $X$ if it is an invariant measure of $X_{t}$ for every $t \in \mathbb{R}$. We will denote by $\mathcal{M}_{X}$ the set of all invariant measures of $X$. A subset $Y \subset Z$ has total probability if for every $\mu \in \mathcal{M}_{X}$ we have $\mu(Y)=1$ (see [15]).

Theorem 1.1. A compact invariant set $\Lambda$ whose singularities are hyperbolic (with ind $\geq$ ind $(\mathcal{J})$ ) for $X \in \mathfrak{X}^{1}(M)$ is a p-singular hyperbolic set if, and only if, there exist a neighborhood $U$ of $\Lambda$ and a field of non-degenerate quadratic forms $\mathcal{J}$ on $U$ with index $1 \leq \operatorname{ind}(\mathcal{J}) \leq n-2$ such that $X$ is non-negative strictly $\mathcal{J}$-separated and the spectrum of the diagonalized operator DX $X_{t}$ satisfies the properties:

(1) $r_{1}^{-}<1$; and

(2) $\Pi_{1}^{p} r_{i}^{+}>1$, where $2 \leq p \leq \operatorname{dim}(M)-\operatorname{ind}(\mathcal{J})$,

in a total probability subset of $\Lambda$. Moreover, if $r_{i}^{+} \cdot r_{j}^{+}>1$, for all $1 \leq i, j, \leq p, i \neq j$, in a total probability set, then $\Lambda$ is a sectional-hyperbolic set. 
In [1], the authors proved the next result about sectional hyperbolicity.

As a direct application of Theorem 1.1 and Theorem2.9 in Section 2, we reobtain the next one, without the assumption on the singularities.

Corollary 1.2. [1, Theorem D] Suppose that all singularities of the attracting set $\Lambda$ of $U$ are all of them sectional-hyperbolic with index $\operatorname{ind}(\sigma) \geq \operatorname{ind}(\Lambda)$. Then, $\Lambda$ is a sectional-hyperbolic set for $X_{t}$ if, and only if, there is a field of quadratic forms $\mathcal{J}$ with index equal to ind $(\Lambda)$ such that $X_{t}$ is a non-negative strictly $\mathcal{J}$-separated flow on $U$ and for each compact invariant subset $\Gamma$ in $\Lambda^{*}=\Lambda \backslash \operatorname{Sing}(X)$ the linear Poincaré flow is strictly $\mathcal{J}_{0}$-monotonous for some field of quadratic forms $\mathcal{J}_{0}$ equivalent to $\mathcal{\partial}$.

Thus, Theorem 1.1 is an improvement to this result, once it does not requires a priori sectional hyperbolicity on the singularities.

In [3], this author together with V. Araujo and A. Arbieto, proved that the requirements in the definition of sectional hyperbolicity can be weakened, demanding the domination property only over the singularities, because in this setting the splitting is in fact dominated. More precisely, we proved the next result.

Theorem 1.3. [3, Theorem A] Let $\Lambda$ be a compact invariant set of $X$ such that every singularity in this set is hyperbolic. Suppose that there exists a continuous invariant splitting of the tangent bundle of $\Lambda, T_{\Lambda} M=E \oplus F$, where $E$ is uniformly contracted, $F$ is sectionally expanding and for some constants $C, \lambda>0$ we have

$$
\left\|\left.D X_{t}\right|_{E_{\sigma}}\right\| \cdot\left\|\left.D X_{-t}\right|_{F_{\sigma}}\right\|<C e^{-\lambda t} \quad \text { for all } \quad \sigma \in \Lambda \cap \operatorname{Sing}(X) \text { and } t \geq 0 .
$$

Then $T_{\Lambda} M=E \oplus F$ is a dominated splitting.

The study of conditions to a given splitting of the tangent bundle to have the domination property is an important research line in the area of Dynamical Systems, see [4], [5], [8].

Some progress in this context has been obtained for instance in [2, Theorem A], jointly with V. Araujo, where we give a characterization for dominated splitting based on $k$-th exterior powers, where $k=\operatorname{dim} F$.

We note that if $E \oplus F$ is a $D X_{t}$-invariant splitting of $T_{\Gamma} M$, with $\left\{e_{1}, \ldots, e_{\ell}\right\}$ a family of basis for $E$ and $\left\{f_{1}, \ldots, f_{h}\right\}$ a family of basis for $F$, then $\widetilde{F}=\wedge^{k} F$ generated by $\left\{f_{i_{1}} \wedge \cdots \wedge\right.$ $\left.f_{i_{k}}\right\}_{1 \leq i_{1}<\cdots<i_{k} \leq h}$ is naturally $\wedge{ }^{k} D X_{t}$-invariant by construction. In addition, $\tilde{E}$ generated by $\left\{e_{i_{1}} \wedge\right.$ $\left.\cdots \wedge e_{i_{k}}\right\}_{1 \leq i_{1}<\cdots<i_{k} \leq \ell}$ together with all the exterior products of $i$ basis elements of $E$ with $j$ basis elements of $F$, where $i+j=k$ and $i, j \geq 1$, is also $\wedge{ }^{k} D X_{t}$-invariant and, moreover, $\widetilde{E} \oplus \widetilde{F}$ gives a splitting of the $k$ th exterior power $\wedge^{k} T_{\Gamma} M$ of the subbundle $T_{\Gamma} M$.

Theorem 1.4. [2, Theorem A] Let $T_{\Gamma} M=E_{\Gamma} \oplus F_{\Gamma}$ be a $D X_{t}$-invariant splitting over the compact $X_{t}$-invariant subset $\Gamma$ such that $\operatorname{dim} F=k \geq 2$. Let $\widetilde{F}=\wedge^{k} F$ be the $\wedge^{k} D X_{t}$-invariant subspace generated by the vectors of $F$ and $\tilde{E}$ be the $\wedge^{k} D X_{t}$-invariant subspace such that $\widetilde{E} \oplus \widetilde{F}$ is a splitting of the kth exterior power $\wedge^{k} T_{\Gamma} M$ of the subbundle $T_{\Gamma} M$.

Then $E \oplus F$ is a dominated splitting if, and only if, $\widetilde{E} \oplus \widetilde{F}$ is a dominated splitting for $\wedge^{k} D X_{t}$.

We note that the equivalence is only valid if $k=\operatorname{dim} F$. 
Here, it is proved a similar result to [3, Theorem A], but now it is done on $p$-sectional hypothesis. Note that, in this case, it is no longer true without some more requirements on the combinations of the Lyapunov exponents of the subbundles (due Theorem 1.4), since for $p>2$ we can have uniform contraction on $E, p$-sectional expansion on $F$ and none dominated splitting, as exemplified below.

In [2, Example 3], we have an example where even $E \oplus F$ being dominated we do not obtain $\widetilde{E} \oplus \widetilde{F}$ dominated, for $k<\operatorname{dim} F$. The next example is a similar one.

Example 1. Theorem 1.4 does not hold if we take $c<\operatorname{dim} F$ : consider $\sigma$ a hyperbolic fixed point for a vector field $X$ in a 4-manifold such that $D X(\sigma)=\operatorname{diag}\{-3,2,4,10\}$. The splitting $E=\mathbb{R} \times\left\{0^{3}\right\}, F=\{0\} \times \mathbb{R}^{3}$ is dominated and hyperbolic but, for $c=2<3=\operatorname{dim} F$ the splitting $\tilde{E} \oplus \tilde{F}$ of the exterior square is not dominated. Indeed, the eigenvalues for $\tilde{F}$ are $2+4=6,2+$ $10=12,4+10=14$, and for $\tilde{E}$ the eigenvalues are $-3+2=-1,-3+4=1,-3+10=7$, so we have an eigenvalue 7 in $\tilde{E}$ strictly bigger than the eigenvalue 6 along $\tilde{F}$.

We can see that even under the domination assumption over singularities, we have no longer the same result as Theorem 1.3, it is enough to take the union of an isolated hyperbolic singularity with a periodic orbit displaying the features of the above example.

However, we might ask how this assumption worked out in [2] and [3]. In fact, within the accounts of the results contained therein it is obtained domination due 2-sectional expansion together with the uniform contraction. The singular case requires domination on the singularities, once it is necessary matching the splitting.

Observing these results, we can get a characterization of domination property based on Lyapunov spectrum, without any other assumption on the singularities. This is the content of our next result.

Theorem 1.5. Let $\Lambda$ be a compact invariant set of $X$. Suppose that there exists a continuous invariant splitting of the tangent bundle of $\Lambda, T_{\Lambda} M=E \oplus F$. Then $T_{\Lambda} M=E \oplus F$ is a dominated splitting if, and only if, exists $\eta<0$ for which

$$
\liminf _{t \rightarrow+\infty} \frac{1}{t} \log \left|D X_{t}\right|_{E_{x}} \mid-\limsup _{t \rightarrow+\infty} \frac{1}{t} \log m\left(\left.D X_{t}\right|_{F_{x}}\right)<\eta,
$$

in a total probability set of $\Lambda$.

By transitivity, we obtain the next corollary.

Corollary 1.6. Suppose the assumptions of Theorem 1.4. Then, $\widetilde{E} \oplus \widetilde{F}$ is a dominated splitting for $\wedge^{k} D X_{t}$ if, and only if, there exists $\eta<0$ for which

$$
\liminf _{t \rightarrow+\infty} \frac{1}{t} \log \left|D X_{t}\right|_{E_{x}} \mid-\limsup _{t \rightarrow+\infty} \frac{1}{t} \log m\left(\left.D X_{t}\right|_{F_{x}}\right)<\eta,
$$

in a total probability set of $\Lambda$.

\section{3. p-sectional Lyapunov exponents.}

The next definition reminds a previous one from Arbieto [6] which deals with, in his terminology, the sectional Lyapunov exponents. 
Based in the same ideas, we can state an analogous term for general singular sets. Inspired by [6], we finally define:

Definition 4. The $p$-sectional Lyapunov exponents (or Lyapunov exponents of order $p$ ) of $x$ along $F$ are the limits

$$
\lim _{t \rightarrow+\infty} \frac{1}{t} \log \left\|\wedge^{p} D X_{t}(x) \cdot \widetilde{v}\right\|
$$

whenever they exists, where $\widetilde{v} \in \wedge^{p} F_{x}-\{0\}$.

Following the corresponding results from [3, Theorem B] and [6, Theorem 2.3], just by some modifications in computations and hyphotesis, changing $\left\|\wedge^{2} D X_{t}(x) . \widetilde{v}\right\|$ by $\left\|\wedge^{p} D X_{t}(x) \cdot \widetilde{v}\right\|$.

We obtain, via Theorem 2.9, the analogous results for singular hyperbolic and partially hyperbolic sets of the main result of this paper.

Corollary 1.7. Let $\Lambda$ be a compact invariant set of $X$ such that every singularity in this set is hyperbolic. There exists a continuous invariant splitting of the tangent bundle, $T_{\Lambda} M=E \oplus F$, of $\Lambda$ where:

(1) the Lyapunov exponents on $E$ are negative (or positive on $F$ ), and

(2) $\liminf _{t \rightarrow+\infty} \frac{1}{t} \log \left|D X_{t}\right|_{E_{x}} \mid-\limsup _{t \rightarrow+\infty} \frac{1}{t} \log m\left(\left.D X_{t}\right|_{F_{x}}\right)<0$,

in a total probability set of $\Lambda$, if and only if, $T_{\Lambda} M=E \oplus F$ is a partially hyperbolic splitting.

This way, we can extend and improve [3, Theorem B] and [6, Theorem 2.3], as follow.

Corollary 1.8. Let $\Lambda$ a compact invariant set for a flow $X_{t}$ such that every singularity $\sigma \in \Lambda$ is hyperbolic. Suppose that there is a continuous invariant splitting $T_{\Lambda} M=E \oplus F$. The set $\Lambda$ is p-singular hyperbolic for the flow if, and only if, on a set of total probability in $\Lambda$,

(1) $\liminf _{t \rightarrow+\infty} \frac{1}{t} \log \left|D X_{t}\right|_{E_{x}} \mid-\limsup _{t \rightarrow+\infty} \frac{1}{t} \log m\left(\left.D X_{t}\right|_{F_{x}}\right)<0$,

(2) the Lyapunov exponents in the E direction are negative and

(3) the p-sectional Lyapunov exponents in the $F$ direction are positive.

Hence, the definition of singular hyperbolicity (of any order, including the classical one) can be rewritten based on the Lyapunov exponents.

Definition 5. A compact invariant set $\Lambda \subset M$ is $p$-singular hyperbolic for $X$ if all singularities in $\Lambda$ are hyperbolic, there are a continuous invariant splitting of the tangent bundle on $T_{\Lambda} M=E \oplus F$ and constants $C, \lambda>0$ such that for every $x \in \Lambda$ and every $t>0$ we have

(1) the Lyapunov exponents in the $E$ direction are negative,

(2) the $p$-sectional Lyapunov exponents in the $F$ direction are positive,

(3) $\liminf _{t \rightarrow+\infty} \frac{1}{t} \log \left|D X_{t}\right|_{E_{x}} \mid-\limsup _{t \rightarrow+\infty} \frac{1}{t} \log m\left(\left.D X_{t}\right|_{F_{x}}\right)<0$

in a total probability set of $\Lambda$.

The last item guarantees that the dominated splitting of the singularities matches to the one over the remainder of $\Lambda$. 
Remark 1.9. The properties of $p$-singular hyperbolicity can be expressed in the following equivalent forms; see [5] for the classical one. There exists $T>0$ such that

- $\left\|\left.D X^{T}\right|_{E_{x}}\right\|<\frac{1}{2}$ for all $x \in \Lambda$ (uniform contraction); and

- $\left|\wedge^{p}\left(D X^{T} \mid \wedge^{p} F_{x}\right)\right|>2$ for all $x \in \Lambda$.

From now on, we consider $M$ a connected compact finite dimensional riemannian manifold and all singularities of $X$ (if they exist) are hyperbolic.

\section{FIELDS OF QUADRATIC FORMS}

In this section, we introduce the quadratic forms and its properties.

Let $\mathcal{J}: E_{U} \rightarrow \mathbb{R}$ be a continuous family of quadratic forms $\mathcal{\partial}_{x}: E_{x} \rightarrow \mathbb{R}$ which are nondegenerate and have index $0<q<\operatorname{dim}(E)=n$, where $U \subset M$ is an open set such that $X_{t}(U) \subset \bar{U}$ for a vector field $X$. We also assume that $\left(\mathcal{J}_{x}\right)_{x \in U}$ is continuously differentiable along the flow.

The continuity assumption on $\mathcal{J}$ just means that for every continuous section $Z$ of $E_{U}$ the map $U \rightarrow \mathbb{R}$ given by $x \mapsto \mathcal{J}(Z(x))$ is continuous. The $C^{1}$ assumption on $\mathcal{J}$ along the flow means that the map $x \mapsto \mathcal{J}_{X_{t}(x)}\left(Z\left(X_{t}(x)\right)\right)$ is continuously differentiable for all $x \in U$ and each $C^{1}$ section $Z$ of $E_{U}$.

The assumption that $M$ is a compact manifold enables us to globally define an inner product in $E$ with respect to which we can find the an orthonormal basis associated to $\partial_{x}$ for each $x$, as follows. Fixing an orthonormal basis on $E_{x}$ we can define the linear operator

$$
J_{x}: E_{x} \rightarrow E_{x} \quad \text { such that } \partial_{x}(v)=<J_{x} v, v>\text { for all } v \in T_{x} M,
$$

where $<,>=<,>_{x}$ is the inner product at $E_{x}$. Since we can always replace $J_{x}$ by $\left(J_{x}+J_{x}^{*}\right) / 2$ without changing the last identity, where $J_{x}^{*}$ is the adjoint of $J_{x}$ with respect to $<,>$, we can assume that $J_{x}$ is self-adjoint without loss of generality. Hence, we represent $\mathcal{J}(v)$ by a nondegenerate symmetric bilinear form $\left\langle\mathcal{\partial}_{x} v, v\right\rangle_{x}$. Now we use Lagrange's method to diagonalize this bilinear form, obtaining a base $\left\{u_{1}, \ldots, u_{n}\right\}$ of $E_{x}$ such that

$$
\partial_{x}\left(\sum_{i} \alpha_{i} u_{i}\right)=\sum_{i=1}^{q}-\lambda_{i} \alpha_{i}^{2}+\sum_{j=q+1}^{n} \lambda_{j} \alpha_{j}^{2}, \quad\left(\alpha_{1}, \ldots, \alpha_{n}\right) \in \mathbb{R}^{n} .
$$

Replacing each element of this base according to $v_{i}=\left|\lambda_{i}\right|^{1 / 2} u_{i}$ we deduce that

$$
\partial_{x}\left(\sum_{i} \alpha_{i} v_{i}\right)=\sum_{i=1}^{q}-\alpha_{i}^{2}+\sum_{j=q+1}^{n} \alpha_{j}^{2}, \quad\left(\alpha_{1}, \ldots, \alpha_{n}\right) \in \mathbb{R}^{n} .
$$

Finally, we can redefine $<,>$ so that the base $\left\{v_{1}, \ldots, v_{n}\right\}$ is orthonormal. This can be done smoothly in a neighborhood of $x$ in $M$ since we are assuming that the quadratic forms are nondegenerate; the reader can check the method of Lagrange in a standard Linear Algebra textbook and observe that the steps can be performed with small perturbations, for instance in [14].

In this adapted inner product we have that $J_{x}$ has entries from $\{-1,0,1\}$ only, $J_{x}^{*}=J_{x}$ and also that $J_{x}^{2}=J_{x}$. 
Having fixed the orthonormal frame as above, the standard negative subspace at $x$ is the one spanned by $v_{1}, \ldots, v_{q}$ and the standard positive subspace at $x$ is the one spanned $v_{q+1}, \ldots, v_{n}$.

2.0.1. Positive and negative cones. Let $\mathcal{C}_{ \pm}=\left\{C_{ \pm}(x)\right\}_{x \in U}$ be the family of positive and negative cones

$$
C_{ \pm}(x):=\{0\} \cup\left\{v \in E_{x}: \pm \mathcal{J}_{x}(v)>0\right\} \quad x \in U
$$

and also let $\mathcal{C}_{0}=\left\{C_{0}(x)\right\}_{x \in U}$ be the corresponding family of zero vectors $C_{0}(x)=\mathcal{J}_{x}^{-1}(\{0\})$ for all $x \in U$. In the adapted coordinates obtained above we have

$$
C_{0}(x)=\left\{v=\sum_{i} \alpha_{i} v_{i} \in E_{x}: \sum_{j=q+1}^{n} \alpha_{j}^{2}=\sum_{i=1}^{q} \alpha_{i}^{2}\right\}
$$

is the set of extreme points of $C_{ \pm}(x)$.

The following definitions are fundamental to state our main result.

Definition 6. Given a continuous field of non-degenerate quadratic forms $\mathcal{J}$ with constant index on the trapping region $U$ for the flow $X_{t}$, we say that the flow is

- J-separated if $D X_{t}(x)\left(C_{+}(x)\right) \subset C_{+}\left(X_{t}(x)\right)$, for all $t>0$ and $x \in U$;

- strictly J-separated if $D X_{t}(x)\left(C_{+}(x) \cup C_{0}(x)\right) \subset C_{+}\left(X_{t}(x)\right)$, for all $t>0$ and $x \in U$;

- J-monotone if $\partial_{X_{t}(x)}\left(D X_{t}(x) v\right) \geq \partial_{x}(v)$, for each $v \in T_{x} M \backslash\{0\}$ and $t>0$;

- strictly $\mathcal{J}$-monotone if $\left.\partial_{t}\left(\mathcal{J}_{X_{t}(x)}\left(D X_{t}(x) v\right)\right)\right|_{t=0}>0$, for all $v \in T_{x} M \backslash\{0\}, t>0$ and $x \in U$;

- J-isometry if $\mathcal{J}_{X_{t}(x)}\left(D X_{t}(x) v\right)=\mathcal{J}_{x}(v)$, for each $v \in T_{x} M$ and $x \in U$.

Thus, $\mathcal{J}$-separation corresponds to simple cone invariance and strict $\mathcal{J}$-separation corresponds to strict cone invariance under the action of $D_{t}(x)$.

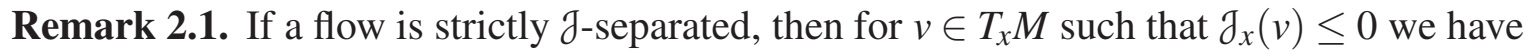

$$
\partial_{X_{-t}(x)}\left(D X_{-t}(v)\right)<0
$$

for all $t>0$ and $x$ such that $X_{-s}(x) \in U$ for every $s \in[-t, 0]$.

Indeed, otherwise $\mathcal{J}_{X_{-t}(x)}\left(D X_{-t}(v)\right) \geq 0$ would imply $\mathcal{J}_{x}(v)=\mathcal{J}_{x}\left(D X_{t}\left(D X_{-t}(v)\right)\right)>0$, contradicting the assumption that $v$ was a non-positive vector.

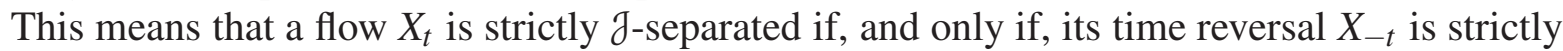
$(-\mathcal{J})$-separated.

A vector field $X$ is $\mathcal{J}$-non-negative on $U$ if $\mathcal{J}(X(x)) \geq 0$ for all $x \in U$, and $\mathcal{J}$-non-positive on $U$ if $\mathcal{J}(X(x)) \leq 0$ for all $x \in U$. When the quadratic form used in the context is clear, we will simply say that $X$ is non-negative or non-positive.

We apply this notion to the linear Poincaré flow defined on regular orbits of $X_{t}$ as follows.

We assume that the vector field $X$ is non-negative on $U$. Then, the span $E_{x}^{X}$ of $X(x) \neq 0$ is a $\mathcal{J}$-non-degenerate subspace. According to item (1) of Proposition 2.3, this means that $T_{x} M=$ $E_{x}^{X} \oplus N_{x}$, where $N_{x}$ is the pseudo-orthogonal complement of $E_{x}^{X}$ with respect to the bilinear form $\mathcal{J}$, and $N_{x}$ is also non-degenerate. Moreover, by the definition, the index of $\mathcal{J}$ restricted to $N_{x}$ is the same as the index of $\mathcal{J}$. Thus, we can define on $N_{x}$ the cones of positive and negative vectors, respectively, $N_{x}^{+}$and $N_{x}^{-}$, just like before. 
Now we define the Linear Poincaré Flow $P^{t}$ of $X_{t}$ along the orbit of $x$, by projecting $D X_{t}$ orthogonally (with respect to $\mathcal{J}$ ) over $N_{X_{t}(x)}$ for each $t \in \mathbb{R}$ :

$$
P^{t} v:=\Pi_{X_{t}(x)} D X_{t} v, \quad v \in T_{x} M, t \in \mathbb{R}, X(x) \neq 0,
$$

where $\Pi_{X_{t}(x)}: T_{X_{t}(x)} M \rightarrow N_{X_{t}(x)}$ is the projection on $N_{X_{t}(x)}$ parallel to $X\left(X_{t}(x)\right)$.

We remark that the definition of $\Pi_{x}$ depends on $X(x)$ and $\mathcal{J}_{X}$ only. The linear Poincare flow $P^{t}$ is a linear multiplicative cocycle over $X_{t}$ on the set $U$ with the exclusion of the singularities of $X$.

In this setting we can say that the linear Poincaré flow is $\mathcal{J}$-separated and $\mathcal{J}$-monotonous using the non-degenerate bilinear form $\mathcal{J}$ restricted to $N_{x}$ for a regular $x \in U$.

More precisely: $P^{t}$ is $\mathcal{J}$-monotonous if $\left.\partial_{t} \mathcal{\partial}\left(P^{t} v\right)\right|_{t=0} \geq 0$, for each $x \in U, v \in T_{x} M \backslash\{0\}$ and $t>0$, and strictly $\mathcal{J}$-monotonous if $\left.\partial_{t} \mathcal{\partial}\left(P^{t} v\right)\right|_{t=0}>0$, for all $v \in T_{x} M \backslash\{0\}, t>0$ and $x \in U$.

Proposition 2.2. Let $L: V \rightarrow V$ be a J-separated linear operator. Then

(1) $L$ can be uniquely represented by $L=R U$, where $U$ is a $\mathcal{J}$-isometry and $R$ is $\mathcal{J}$-symmetric (or J-pseudo-adjoint; see Proposition 2.3) with positive spectrum.

(2) the operator $R$ can be diagonalized by a J-isometry. Moreover the eigenvalues of $R$ satisfy

$$
0<r_{-}^{q} \leq \cdots \leq r_{-}^{1}=r_{-} \leq r_{+}=r_{1}^{+} \leq \cdots \leq r_{+}^{p} .
$$

(3) the operator L is (strictly) J-monotonous if, and only if, $r_{-} \leq(<) 1$ and $r_{+} \geq(>) 1$.

\subsection{J-separated linear maps.}

2.1.1. J-symmetrical matrixes and J-selfadjoint operators. The symmetrical bilinear form defined by

$$
(v, w)=\left\langle J_{x} v, w\right\rangle,
$$

$v, w \in E_{x}$ for $x \in M$ endows $E_{x}$ with a pseudo-Euclidean structure.

Since $\mathcal{J}_{x}$ is non-degenerate, then the form $(\cdot, \cdot)$ is likewise non-degenerate and many properties of inner products are shared with symmetrical non-degenerate bilinear forms. We state some of them below.

Proposition 2.3. Let $(\cdot, \cdot): V \times V \rightarrow \mathbb{R}$ be a real symmetric non-degenerate bilinear form on the real finite dimensional vector space $V$.

(1) $E$ is a subspace of $V$ for which $(\cdot, \cdot)$ is non-degenerate if, and only if, $V=E \oplus E^{\perp}$.

We recall that $E^{\perp}:=\{v \in V:(v, w)=0$ for all $w \in E\}$, the pseudo-orthogonal space of $E$, is defined using the bilinear form.

(2) Every base $\left\{v_{1}, \ldots, v_{n}\right\}$ of $V$ can be orthogonalized by the usual Gram-Schmidt process of Euclidean spaces, that is, there are linear combinations of the basis vectors $\left\{w_{1}, \ldots, w_{n}\right\}$ such that they form a basis of $V$ and $\left(w_{i}, w_{j}\right)=0$ for $i \neq j$. Then this last base can be pseudo-normalized: letting $u_{i}=\left|\left(w_{i}, w_{i}\right)\right|^{-1 / 2} w_{i}$ we get $\left(u_{i}, u_{j}\right)= \pm \delta_{i j}, i, j=1, \ldots, n$.

(3) There exists a maximal dimension $p$ for a subspace $P_{+}$of $\mathcal{J}$-positive vectors and a maximal dimension $q$ for a subspace $P_{-}$of $\mathcal{J}$-negative vectors; we have $p+q=\operatorname{dim} V$ and $q$ is known as the index of $\mathcal{J}$. 
(4) For every linear map $L: V \rightarrow \mathbb{R}$ there exists a unique $v \in V$ such that $L(w)=(v, w)$ for each $w \in V$.

(5) For each $L: V \rightarrow V$ linear there exists a unique linear operator $L^{+}: V \rightarrow V$ (the pseudoadjoint) such that $(L(v), w)=\left(v, L^{+}(w)\right)$ for every $v, w \in V$.

(6) Every pseudo-self-adjoint $L: V \rightarrow V$, that is, such that $L=L^{+}$, satisfies

(a) eigenspaces corresponding to distinct eigenvalues are pseudo-orthogonal;

(b) if a subspace $E$ is L-invariant, then $E^{\perp}$ is also L-invariant.

The proofs are rather standard and can be found in [14].

The following simple result will be very useful in what follows.

Lemma 2.4. Let $V$ be a real finite dimensional vector space endowed with a non-positive definite and non-degenerate quadratic form $\mathcal{J}: V \rightarrow \mathbb{R}$.

If a symmetric bilinear form $F: V \times V \rightarrow \mathbb{R}$ is non-negative on $C_{0}$ then

$$
r_{+}=\inf _{v \in C_{+}} \frac{F(v, v)}{\langle J v, v\rangle} \geq \sup _{u \in C_{-}} \frac{F(u, u)}{\langle J u, u\rangle}=r_{-}
$$

and for every $r$ in $\left[r_{-}, r_{+}\right]$we have $F(v, v) \geq r\langle J v, v\rangle$ for each vector $v$.

In addition, if $F(\cdot, \cdot)$ is positive on $C_{0} \backslash\{0\}$, then $r_{-}<r_{+}$and $F(v, v)>r\langle J v, v\rangle$ for all vectors $v$ and $r \in\left(r_{-}, r_{+}\right)$.

Remark 2.5. Lemma 2.4 shows that if $F(v, w)=\langle\tilde{J} v, w\rangle$ for some self-adjoint operator $\tilde{J}$ and $F(v, v) \geq 0$ for all $v$ such that $\langle J v, v\rangle=0$, then we can find $a \in \mathbb{R}$ such that $\tilde{J} \geq a J$. This means precisely that $\langle\tilde{J} v, v\rangle \geq a\langle J v, v\rangle$ for all $v$.

If, in addition, we have $F(v, v)>0$ for all $v$ such that $\langle J v, v\rangle=0$, then we obtain a strict inequality $\tilde{J}>a J$ for some $a \in \mathbb{R}$ since the infimum in the statement of Lemma 2.4 is strictly bigger than the supremum.

The (longer) proofs of the following results can be found in [25] or in [21]; see also [26].

For a $\mathcal{J}$-separated operator $L: V \rightarrow V$ and a $d$-dimensional subspace $F_{+} \subset C_{+}$, the subspaces $F_{+}$and $L\left(F_{+}\right) \subset C_{+}$have an inner product given by $\mathcal{J}$. Thus both subspaces are endowed with volume elements. Let $\alpha_{d}\left(L ; F_{+}\right)$be the rate of expansion of volume of $\left.L\right|_{F_{+}}$and $\sigma_{d}(L)$ be the infimum of $\alpha_{d}\left(L ; F_{+}\right)$over all $d$-dimensional subspaces $F_{+}$of $C_{+}$.

Proposition 2.6. We have $\sigma_{d}(L)=r_{+}^{1} \cdots r_{+}^{d}$, where $r_{+}^{i}$ are given by Proposition 2.2(2).

Moreover, if $L_{1}, L_{2}$ are $\mathcal{J}$-separated, then $\sigma_{d}\left(L_{1} L_{2}\right) \geq \sigma_{d}\left(L_{1}\right) \sigma_{d}\left(L_{2}\right)$.

The following corollary is very useful.

Corollary 2.7. For J-separated operators $L_{1}, L_{2}: V \rightarrow V$ we have

$$
r_{+}^{1}\left(L_{1} L_{2}\right) \geq r_{+}^{1}\left(L_{1}\right) r_{+}^{1}\left(L_{2}\right) \quad \text { and } \quad r_{-}^{1}\left(L_{1} L_{2}\right) \leq r_{-}^{1}\left(L_{1}\right) r_{-}^{1}\left(L_{2}\right) .
$$

Moreover, if the operators are strictly J-separated, then the inequalities are strict.

Remark 2.8. Another important property about the singular values of a $\mathcal{J}$-separated operator $L$ is that

$$
r_{+}^{1}=r_{+} \geq 1(>1) \quad \text { and } \quad r_{-}^{1}=r_{-} \leq 1(<1)
$$


if, and only if, $L$ is (strictly) $\mathcal{J}$-monotone.

This property will be used a lot of times in our proofs.

\subsection{Lyapunov exponents.}

It is well known that under conditions of measurability, by Oseledec's Ergodic Theorem, there exist a full probability set $X$ such that for every $x \in Y$ there is an invariant decomposition

$$
T_{x} M=\langle X\rangle \oplus E_{1}(x) \oplus \cdots \oplus E_{l(x)}(x)
$$

and numbers $\chi_{1}<\cdots<\chi_{l}$ corresponding to the limits

$$
\chi_{j}=\lim _{t \rightarrow+\infty} \frac{1}{t} \log \left\|D X_{t}(x) \cdot v\right\|
$$

for every $v \in E_{i}(x) \backslash\{0\}, i=1, \cdots, l(x)$.

In this setting, Wojtkowski [25] proved that the logarithm of the pseudo-Euclidean singular values $0 \leq r_{q}^{-} \leq \cdots \leq r_{1}^{-} \leq r_{1}^{+} \leq \cdots \leq r_{p}^{+}$of $D X_{t}$ are $\mu$-integrable, and obtained estimates of the Lyapunov exponents related to the singular eigenvalues of strictly $\mathcal{J}$-separated maps.

Theorem 2.9. [25, Corollary 3.7]

For $1 \leq k_{1} \leq p$ and $1 \leq k_{2} \leq q$

$$
\chi_{1}^{-}+\cdots+\chi_{k_{1}}^{-} \leq \sum_{i=1}^{k_{1}} \int \log r_{i}^{-} d \mu \text { and } \chi_{1}^{+}+\cdots+\chi_{k_{2}}^{+} \geq \sum_{i=1}^{k_{2}} \int \log r_{i}^{+} d \mu \text {. }
$$

Look that, if $X_{t}$ is a $\mathcal{J}$-separated flow on $\Lambda$, for each diffeomorphism $D X_{t}$ if we fix $t>0$, the last theorem holds for $r_{i}^{ \pm, t}$, where $r_{i}^{ \pm, t}$ are the singular $\mathcal{J}$-values of $D X_{t}$.

\section{PROOF OF THEOREMS}

In this section, we prove our mains results.

First, we prove Theorem 1.1, by using Corollary 1.8 which is proved below.

Proof of Theorem 1.1. Suppose $\Lambda p$-singular hyperbolic set of index ind. Then, $1 \leq$ ind $\leq n-2$ and there is a dominated splitting $T_{\Lambda} M=E \oplus F$, where $E$ is uniformly contracting and $F$ is uniformly $p$-sectionally expanding. Moreover, $\langle X\rangle \subset F$, by Lemma 3.2. By using adapted metric [9], we construct the quadratic forms $\mathcal{J}$ such that $X$ is non-negative strictly $\mathcal{J}$-separated. By Proposition 2.2 and Corollary 2.7, there is a $\mathcal{J}$-diagonalization of $D X_{t}$ by a $\mathcal{J}$-isometry, that we are also denoting by $D X_{t}$, such that its spectrum has the required properties. In fact, for each singular value $r_{i}^{-}$corresponding to the contracting subspace, we must have $r_{i}^{-}<1$. Analogously, as $F$ is a $p$-sectionally expanding subbundle, the sum of each $p$ corresponding singular value, $r_{i_{1}}^{+}, \cdots, r_{i_{p}}^{+}$, must be greater than one. Even including the corresponding field direction.

Reciprocally, suppose that in a total probability subset of $\Lambda$ we have $r_{1}^{-}<1$ and $\Pi_{j=1}^{p} r_{i_{j}}^{+}>1$, where $2 \leq p \leq \operatorname{dim}(M)-\operatorname{ind}(\mathcal{J})$. 
Moreover, strictly $\mathcal{J}$-separation guarantees that there exists a dominated splitting. Let $T_{\Lambda} M=$ $E \oplus F$ the corresponding splitting and the decomposition in direct sum of Lyapunov subspaces

$$
E_{x}=\oplus_{j=0}^{r} E_{j}(x), F_{x}=\oplus_{j=0}^{s(x)-1} F_{j}(x) .
$$

By Theorem 2.9,

$$
\chi_{1}^{-}+\cdots+\chi_{r}^{-} \leq \sum_{i=1}^{r} \int \log r_{i}^{-} d \mu \text { and } \chi_{i_{0}}^{+}+\cdots+\chi_{i_{p}}^{+} \geq \sum_{j=1}^{p} \int \log r_{i_{j}}^{+} d \mu .
$$

So, we obtain that the Lyapunov exponents over $E$ are all of them negative and the $p$-sectional Lyapunov exponents on $F$ are all of them positive, in a total probability subset. Now, Theorem 1.5 and Corollary 1.8 imply that $\Lambda$ is a $p$-singular hyperbolic set for $X$.

We recall now that, fixed a compact $X_{t}$-invariant subset $\Lambda$, we say that a family of functions $\left\{f_{t}: \Lambda \rightarrow \mathbb{R}\right\}_{t \in \mathbb{R}}$ is subadditive if for every $x \in M$ and $t, s \in \mathbb{R}$ we have that $f_{t+s}(x) \leq f_{s}(x)+$ $f_{t}\left(X_{S}(x)\right)$.

Proof of Theorem 1.5. Note that, once $T_{\Lambda} M=E \oplus F$ is a dominated splitting, there is an indefinite $C^{1}$ field of quadratic forms $\mathcal{J}$ such a way $X$ is strictly separated and, by Proposition 2.2,

$$
0<r_{-}^{q} \leq \cdots \leq r_{-}^{1}=r_{-}<r_{+}=r_{1}^{+} \leq \cdots \leq r_{+}^{p} .
$$

Moreover, by Corollary 2.9,

$$
\chi_{1}^{-}+\cdots+\chi_{k_{1}}^{-} \leq \sum_{i=1}^{k_{1}} \int \log r_{i}^{-} d \mu \text { and } \chi_{1}^{+}+\cdots+\chi_{k_{2}}^{+} \geq \sum_{i=1}^{k_{2}} \int \log r_{i}^{+} d \mu .
$$

Since $r_{-}-r_{+}<0$, we obtain

$$
\begin{array}{r}
\liminf _{t \rightarrow+\infty} \frac{1}{t} \log \left|D X_{t}\right|_{E_{x}} \mid-\limsup _{t \rightarrow+\infty} \frac{1}{t} \log m\left(\left.D X_{t}\right|_{F_{x}}\right)= \\
=\max \left\{\chi_{i}^{E}(x), 1 \leq i \leq r(x)\right\}-\min \left\{\chi_{i}^{F}(x), 1 \leq i \leq s(x)\right\} \leq \eta<0,
\end{array}
$$

for all $x \in \Lambda$, in particular, in a total probability set.

Reciprocally, suppose that there exists a continuous invariant decomposition, $T_{\Lambda} M=E \oplus F$, and $\eta<0$ such that

$$
\liminf _{t \rightarrow+\infty} \frac{1}{t} \log \left|D X_{t}\right|_{E_{x}} \mid-\limsup _{t \rightarrow+\infty} \frac{1}{t} \log m\left(\left.D X_{t}\right|_{F_{x}}\right) \leq \eta<0
$$

in a total probability set in $\Lambda$.

Consider $f_{t}(x)=\log \frac{\left\|D X_{t} \mid E_{x}\right\|}{m\left(D X_{t} \mid F_{x}\right)}$, which is a subadditive family of continuous functions and satisfies

$$
\bar{f}(x)=\liminf _{t \rightarrow+\infty} \frac{f_{t}(x)}{t} \leq \liminf _{n \rightarrow+\infty} \frac{1}{t} \log \left\|D X_{t} \mid E_{x}\right\|-\limsup _{n \rightarrow+\infty} \frac{1}{t} \log m\left(D X_{t} \mid F_{x}\right) \leq \eta<0 .
$$


By Subadittive Ergodic Theorem [12], the function $\bar{f}(x)=\liminf _{t \rightarrow+\infty} \frac{f_{t}(x)}{t}$ coincides with $\widetilde{f}(x)=$ $\lim _{t \rightarrow+\infty} \frac{1}{t} f_{t}(x)$ in a set of total probability. Moreover, for any invariant measure $\mu$ we have that $\int \widetilde{f} d \mu=\lim _{t \rightarrow+\infty} \int \frac{f_{t}}{t} d \mu$.

Thus, we can use the following result from [4].

Proposition 3.1. [4, Corollary 4.2] Let $\left\{t \mapsto f_{t}: S \rightarrow \mathbb{R}\right\}_{t \in \mathbb{R}}$ be a continuous family of continuous functions which is subadditive and suppose that $\int \widetilde{f}(x) d \mu<0$ for every $\mu \in \mathcal{M}_{X}$, with $\widetilde{f}(x):=$ $\lim _{t \rightarrow+\infty} \frac{1}{t} f_{t}(x)$. Then there exist a $T>0$ and a constant $\eta<0$ such that for every $x \in S$ and every $t \geq T$ :

$$
f_{t}(x) \leq \eta t
$$

Note that, all of the last accounts are true independently to $x$ is either a regular or a singular point.

Hence, we obtain $f_{t}(x) \leq k-\eta t, t \geq 0, x \in \Lambda$, for some constant $k>0$, and this gives us the domination property on $\Lambda$.

Now, we prove the Corollary 1.7.

Proof of Corollary 1.7. Suppose that we are under the hypothesis.

By Theorem 1.5, $E \oplus F$ is a dominated splitting on $\Lambda$.

Since $E$ is an invariant subbundle, consider $f_{t}(x)=\log \left\|\left.D X_{t}\right|_{E_{x}}\right\|, t \in \mathbb{R}$, as our subadditive family.

As in the proof of Theorem 1.5, we obtain $f_{t}(x) \leq k-\eta t, t \geq 0, x \in \Lambda$, for some constant $k>0$. This means that $E$ is uniformly contracting under the action of $D X_{t}$.

The case of positive Lyapunov exponents over $F$ is analogous, by taking $f_{t}(x)=\log \|\left. D X_{-t}\right|_{F_{x}}$. (Also see proof of [3, Theorem B]).

For the converse, by using adapted metrics (as in the proof [1, Theorem A]) we obtain a $C^{1}$ field $\mathcal{J}$ of nondegenerate quadratic forms for which $X$ is nonnegative strictly separated. Now, Proposition 2.2 and Theorem 2.9 complete the proof.

Finally, the proof of Corollary 1.8.

We also need to use the following lemma.

Let $\Lambda$ be a compact invariant set for a flow $X$ of a $C^{1}$ vector field $X$ on $M$.

Lemma 3.2. [3] Given a continuous splitting $T_{\Lambda} M=E \oplus F$ such that $E$ is uniformly contracted, then $X(x) \in F_{x}$ for all $x \in \Lambda$.

Proof of Corollary 1.8. By Theorem 1.5, $T_{\Lambda} M=E \oplus F$ is a dominated splitting.

If $x=\sigma \in \operatorname{Sing}(X)$, by hyperbolicity, we obtain the desired features.

Following Corollary 1.7 , we obtain that this is a partially hyperbolic splitting as well, with subbundle $E$ uniformly contracting. By Lemma 3.2, if $x$ is a regular point, the flow direction $E^{X}(x)$ is contained in $F(x)$. 
Since $F$ is an invariant subbundle, consider $f_{t}(x)=\log \left\|\left.\wedge^{p} D X_{t}\right|_{F_{x}}\right\|, t \in \mathbb{R}$, and there is a decomposition in direct sum of Lyapunov subspaces

$$
F_{x}=\oplus_{j=0}^{s(x)-1} F_{j}(x) .
$$

One of them, say $E^{X}=F_{0}(x)$, generated by $X(x) \neq 0$. Denote by $\chi_{j}^{F}(x), j=1, \cdots s(x)-1$ the corresponding Lyapunov exponents.

Fixing $i_{1}, \cdots, i_{p} \in\{1, \cdots, s(x)-1\}$ and considering $p$ vectors $v_{1} \in F_{i_{1}} \backslash\{0\}, \cdots v_{p-1} \in F_{i_{p-1}} \backslash$ $\{0\}$, put $L=\operatorname{span}\left\{X(x), v_{1}, \cdots, v_{p-1}\right\}$ as the generated $p$-plane.

From assumption,

$$
0<\chi \leq \liminf _{t \rightarrow+\infty} \frac{1}{t} \log \left|\wedge^{p} D X_{t}\right|_{L} \mid=\chi_{0}^{F}+\chi_{i_{1}}^{F}+\cdots+\chi_{i_{p-1}}^{F},
$$

and we obtain

$$
\sum_{j=1}^{p} \chi_{i_{j}}^{F} \geq \chi>0, \forall i_{j} \in\{1, \cdots, s(x)-1\} .
$$

For some singularity, $\sigma \in \Lambda$, we must have $\overline{f(\sigma)} \leq-\chi$, as a consequence of domination . Now applying the following proposition from [6]:

Proposition 3.3. Let $\left\{t \mapsto f_{t}: \Lambda \rightarrow \mathbb{R}\right\}_{t \in \mathbb{R}}$ be a continuous family of continuous function which is subadditive and suppose that $\bar{f}(x)<0$ in a set of total probability. Then there exist constants $C>0$ and $\lambda<0$ such that for every $x \in \Lambda$ and every $t>0$ we have $\exp \left(f_{t}(x)\right) \leq C \exp \left(\frac{\lambda t}{2}\right)$,

to the function $f_{t}(x)$ give us constants $D>0$ and $\eta<0$ for which $\left\|\wedge^{p} D X_{-t} \mid \wedge^{p} F_{X_{t}(x)}\right\| \leq D e^{\eta t}$, so $F$ is a $p$-sectionally expanding subbundle.

The converse follows from the lines of the last proof, by using Proposition 2.2 and Theorem 2.9. So, we are done.

\section{REFERENCES}

1. V. Araujo and L. Salgado. Infinitesimal Lyapunov functions for singular flows. Mathematische Zeitschrift, 275(3-4):863-897, 2013.

2. V. Araújo, L. S. Salgado. Dominated splittings for exterior powers and singular hyperbolicity. J. Differential Equations, 259, 3874-3893. 2015.

3. V. Araujo, A. Arbieto, and L. Salgado. Dominated splittings for flows with singularities. Nonlinearity, 26, 2391-2407. 2013.

4. A. Arbieto and L. Salgado. On critical orbits and sectional hyperbolicity of the nonwandering set for flows. Journal of Differential Equations, 250:2927-2939, 2011.

5. V. Araújo and M. J. Pacifico. Three-dimensional flows, volume 53 of Ergebnisse der Mathematik und ihrer Grenzgebiete. 3. Folge. A Series of Modern Surveys in Mathematics [Results in Mathematics and Related Areas. 3rd Series. A Series of Modern Surveys in Mathematics]. Springer, Heidelberg, 2010. With a foreword by Marcelo Viana.

6. A. Arbieto. Sectional lyapunov exponents. Proc. of the Amercian Mathematical Society, 138:3171-3178, 2010.

7. L., Arnold. Random Dynamical Systems. Springer-Verlag. 2003. 
8. C. Bonatti, L. J. Díaz, and M. Viana. Dynamics beyond uniform hyperbolicity, volume 102 of Encyclopaedia of Mathematical Sciences. Springer-Verlag, Berlin, 2005. A global geometric and probabilistic perspective, Mathematical Physics, III.

9. N. Gourmelon. Adapted metrics for dominated splittings. Ergodic Theory Dynam. Systems, 27(6):1839-1849, 2007.

10. M. Hirsch, C. Pugh, and M. Shub. Invariant manifolds, volume 583 of Lect. Notes in Math. Springer Verlag, New York, 1977.

11. A. Katok. Infinitesimal Lyapunov functions, invariant cone families and stochastic properties of smooth dynamical systems. Ergodic Theory Dynam. Systems, 14(4):757-785, 1994. With the collaboration of Keith Burns.

12. J.F.C. Kingman. The ergodic theory of subadditive stochastic processes. J. Roy.Statist. Soc. Ser. B, 30, 499-510, 1968.

13. J. Lewowicz. Lyapunov functions and topological stability. J. Differential Equations, 38(2):192-209, 1980.

14. A. I. Mal'cev. Foundations of linear algebra. Translated from the Russian by Thomas Craig Brown; edited by J. B. Roberts. W. H. Freeman \& Co., San Francisco, Calif.-London, 1963.

15. R. Mañé. An ergodic closing lemma. Annals of Math., 116:503-540, 1982.

16. R. Metzger and C. Morales. Sectional-hyperbolic systems. Ergodic Theory and Dynamical System, 28:15871597, 2008.

17. C. A. Morales, M. J. Pacifico, and E. R. Pujals. Singular hyperbolic systems. Proc. Amer. Math. Soc., 127(11):3393-3401, 1999.

18. C. A. Morales, M. J. Pacifico, and E. R. Pujals. Robust transitive singular sets for 3-flows are partially hyperbolic attractors or repellers. Ann. of Math. (2), 160(2):375-432, 2004.

19. V. I. Oseledec. A multiplicative ergodic theorem: Lyapunov characteristic numbers for dynamical systems. Trans. Moscow Math. Soc., 19:197-231, 1968.

20. V. P. Potapov. The multiplicative structure of $J$-contractive matrix functions. Amer. Math. Soc. Transl. (2), 15:131-243, 1960. Translation of Trudy Moskovskogo Matematičeskogo Obščestva 4 (1955), 125-236.

21. V. P. Potapov. Linear-fractional transformations of matrices. In Studies in the theory of operators and their applications (Russian), pages 75-97, 177. "Naukova Dumka", Kiev, 1979.

22. P. Walters. An introduction to ergodic theory. Springer Verlag, 1982.

23. M. Wojtkowski. Invariant families of cones and Lyapunov exponents. Ergodic Theory Dynam. Systems, 5(1):145-161, 1985.

24. M. P. Wojtkowski. Magnetic flows and Gaussian thermostats on manifolds of negative curvature. Fund. Math., 163(2):177-191, 2000.

25. M. P. Wojtkowski. Monotonicity, $J$-algebra of Potapov and Lyapunov exponents. In Smooth ergodic theory and its applications (Seattle, WA, 1999), volume 69 of Proc. Sympos. Pure Math., pages 499-521. Amer. Math. Soc., Providence, RI, 2001.

26. M. P. Wojtkowski. A simple proof of polar decomposition in pseudo-Euclidean geometry. Fund. Math., 206:299-306, 2009.

Universidade Federal da Bahia, Instituto de Matemática, Avenida Adhemar de Barros, s/N, ONDINA, 40170-110, SALVADOR, BAHIA, BRAZIL, EMAIL: LSALGADO@UFBA.BR, LSALGADo@IM.UFRJ.BR 